\title{
Schizencephaly and Porencephaly Due to Fetal Intracranial Hemorrhage: A Report of Two Cases
}

\author{
Takashi Harada, Takashi Uegaki, Kazuya Arata, Takako Tsunetou, Fuminori Taniguchi and Tasuku Harada
}

Division of Reproductive-Perinatal Medicine and Gynecologic Oncology, Department of Surgery, School of Medicine, Tottori University Faculty of Medicine, Yonago 683-8503, Japan

\begin{abstract}
Schizencephaly and porencephaly are extremely rare types of cortical dysplasia. Case 1: Prenatal magnetic resonance imaging (MRI) showed wide clefts in the frontal and parietal lobes bilaterally. On postnatal day 3, MRI T2-weighted images showed multiple hypointensities in the clefts and ventricular walls, suggestive of hemosiderosis secondary to intracranial hemorrhage. Case 2: Prenatal MRI showed bilateral cleft and cyst formation in the fetal cerebrum, as well as calcification and hemosiderosis indicative of past hemorrhage. T2-weighted images showed hypointensities in the same regions as the calcification, corresponding with hemosiderosis due to intracranial hemorrhage on postnatal day 10. Thus, prenatal MRI was useful for diagnosing schizencephaly and porencephaly. Schizencephaly and porencephaly were thought to be due to fetal intracranial hemorrhage, which, in the porencephaly case, may have been related to a mutation of COLAAI.
\end{abstract}

Key words fetal intracranial hemorrhage; porencephaly; schizencephaly; ventriculomegaly

Schizencephaly is a type of cortical dysplasia characterized by the formation of clefts in the cerebral mantle. Since these clefts result from destructive changes arising prior to the completion of neuronal formation and migration, they are lined with abnormal gray matter. ${ }^{1-3}$ Conversely, porencephaly, which is characterized by cystic changes in the cerebral parenchyma, results from destructive changes occurring after the completion of neuronal migration; thus, the cyst walls and clefts are not lined with gray matter ${ }^{3,4}$ In both disorders, postnatal clinical symptoms may include various degrees of cerebral palsy, mental retardation, and epilepsy. The clinical

Corresponding author: Takashi Harada, MD, $\mathrm{PhD}$

tkharada@med.tottori-u.ac.jp

Received 2017 September 1

Accepted 2017 October 30

Abbreviations: COL4A1, collagen type IV alpha 1 chain; CMV, cytomegalovirus; CT, computed tomography; GA, gestational age; MRI, magnetic resonance imaging symptoms of schizencephaly depend on the size of the clefts. ${ }^{5}$ Cytomegalovirus (CMV) infection and neuronal migration disorder have long been among the suspected causes of both disorders. Fetal intracranial hemorrhage caused by abnormal type IV collagen, which is a component of vascular walls, has recently been presented as evidence for another etiology. ${ }^{6}$

Two cases, one of schizencephaly and one of porencephaly, both attributed to fetal intracranial bleeding, are reported herein.

\section{PATIENT REPORT \\ Case 1}

Transabdominal ultrasound examination at a gestational age (GA) of 24 weeks 6 days in a 24-year-old Japanese pregnant woman (gravida 3, para 2) indicated fetal ventriculomegaly. The patient was referred to our department at 27 weeks 2 days GA. She had received prenatal checkups from her local doctor, and her family and medical histories were unremarkable. Maternal blood testing for CMV was positive for immunoglobulin (Ig) $\mathrm{G}$ antibodies and negative for IgM antibodies. Clefts in the fetal parietal lobe observed on transabdominal ultrasound suggested schizencephaly (Fig. 1A). Subsequent observation of bilateral wide clefts in the frontal and parietal lobes on magnetic resonance imaging (MRI) at a GA of 30 weeks 0 days led to a prenatal diagnosis of open-lip schizencephaly (Figs. 1B and C). No other associated malformations were observed on prenatal MRI and ultrasound examination.

A 3168-g girl was delivered vaginally at 38 weeks 6 days GA. On postnatal day 3, MRI demonstrated continuous gray matter extending from the lateral ventricles to the brain surface in bilateral clefts (Fig. 1D). T2-weighted images showed multiple hypointensities in the clefts and ventricular walls, suggestive of hemosiderosis secondary to intracranial hemorrhage (Figs. 1E and F). A neonatal blood sample was negative for CMV DNA by PCR. We did not investigate the genetic testing for the COL4A1 mutation. The infant could feed by mouth and was discharged on postnatal day 6 . 

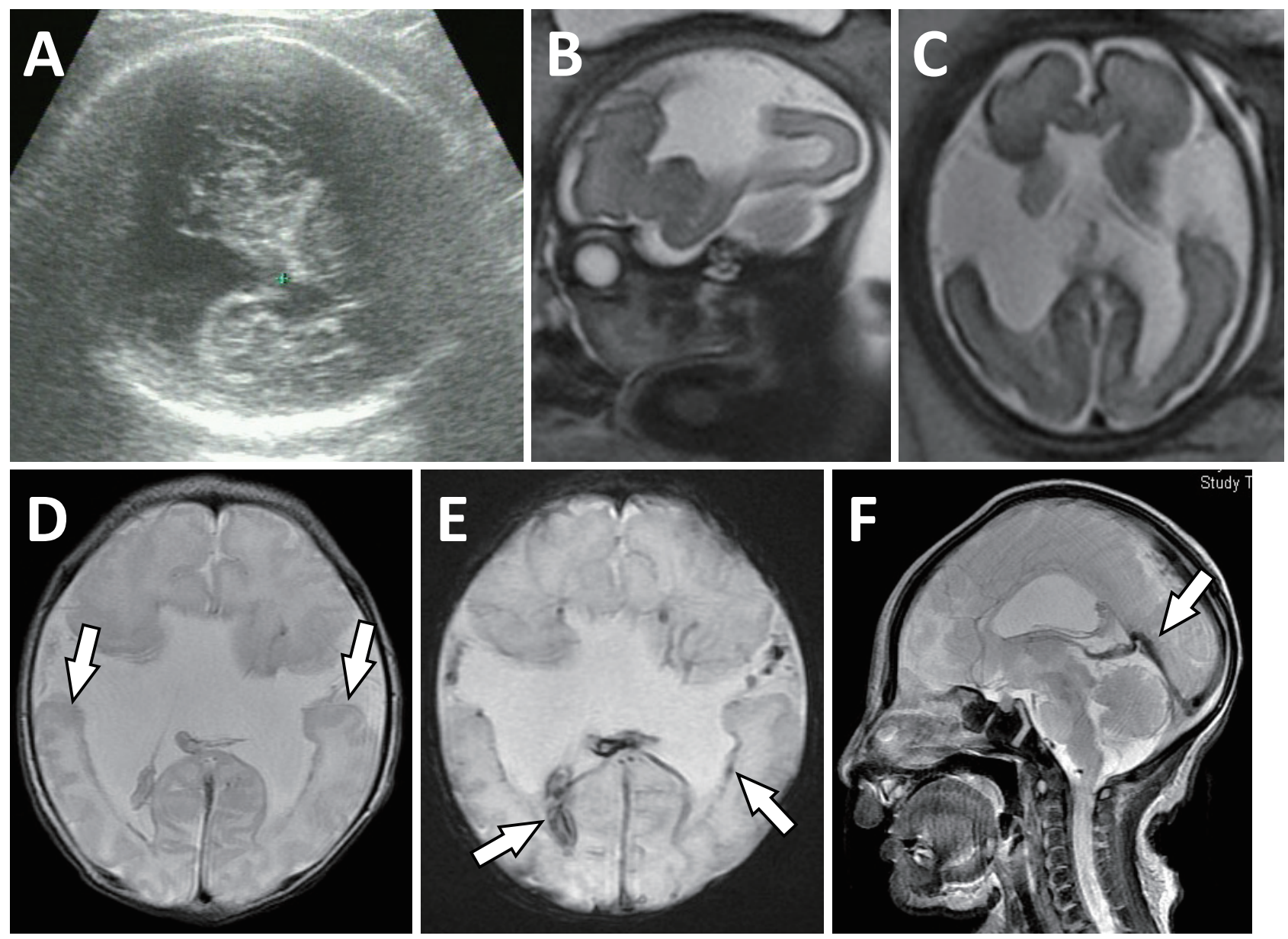

Fig. 1. Prenatal imaging findings and postnatal magnetic resonance imaging of Case 1. A, B, C: Prenatal transabdominal ultrasound and magnetic resonance images. A, Clefts in the parietal lobe, as seen on transabdominal ultrasound at 27 weeks 2 days. B, C: Wide clefts in bilateral frontal and parietal lobes, as seen on magnetic resonance imaging (T2-weighted images) at 30 weeks 0 days. D, E, F: Postnatal magnetic resonance images. D, Clefts in bilateral frontal and parietal lobes lined with continuous gray matter extending from the lateral ventricles to the brain surface, as seen on magnetic resonance imaging (T2-weighted images) on postnatal day 3 (arrows). E, F: Multiple hypointensities are evident on the T2-weighted images (arrows).

\section{Case 2}

Transabdominal ultrasound examination at a GA of 30 weeks 4 days in a 39-year-old Japanese pregnant woman (gravida 1, para 0) indicated fetal ventriculomegaly, and the patient was referred to a general hospital. She had received prenatal care from her local doctor, and her family and medical histories were unremarkable. MRI at a GA of 32 weeks 0 days revealed bilateral clefts and cysts in the fetal cerebrum, as well as calcification and hemosiderosis indicative of past hemorrhage, which led to a prenatal diagnosis of porencephaly (Figs. 2A and $\mathrm{B})$. The patient was referred to our department for delivery at 36 weeks 5 days GA. At initial examination, transabdominal ultrasound confirmed fetal ventriculomegaly (Fig. 2C). No other associated malformations were observed on prenatal MRI or ultrasound. A 1834-g girl was delivered vaginally at 38 weeks 0 days GA. The Apgar score was 5 points at one minute after birth and five-minute score was 7 points.

Destructive changes in the neonatal cerebrum were observed on MRI and computed tomography (CT) on postnatal day 10, confirming the diagnosis of porencephaly. Periventricular calcifications were also observed around the lateral ventricles on CT (Fig. 2D). T2-weighted images showed hypointensities in the same regions as the calcification, corresponding with hemosiderosis due to intracranial hemorrhage (Figs. 2E and F). Urine collected from the infant on postnatal day 12 was negative for CMV by quantitative real-time PCR. Genetic testing identified a heterozygous missense mutation in the 

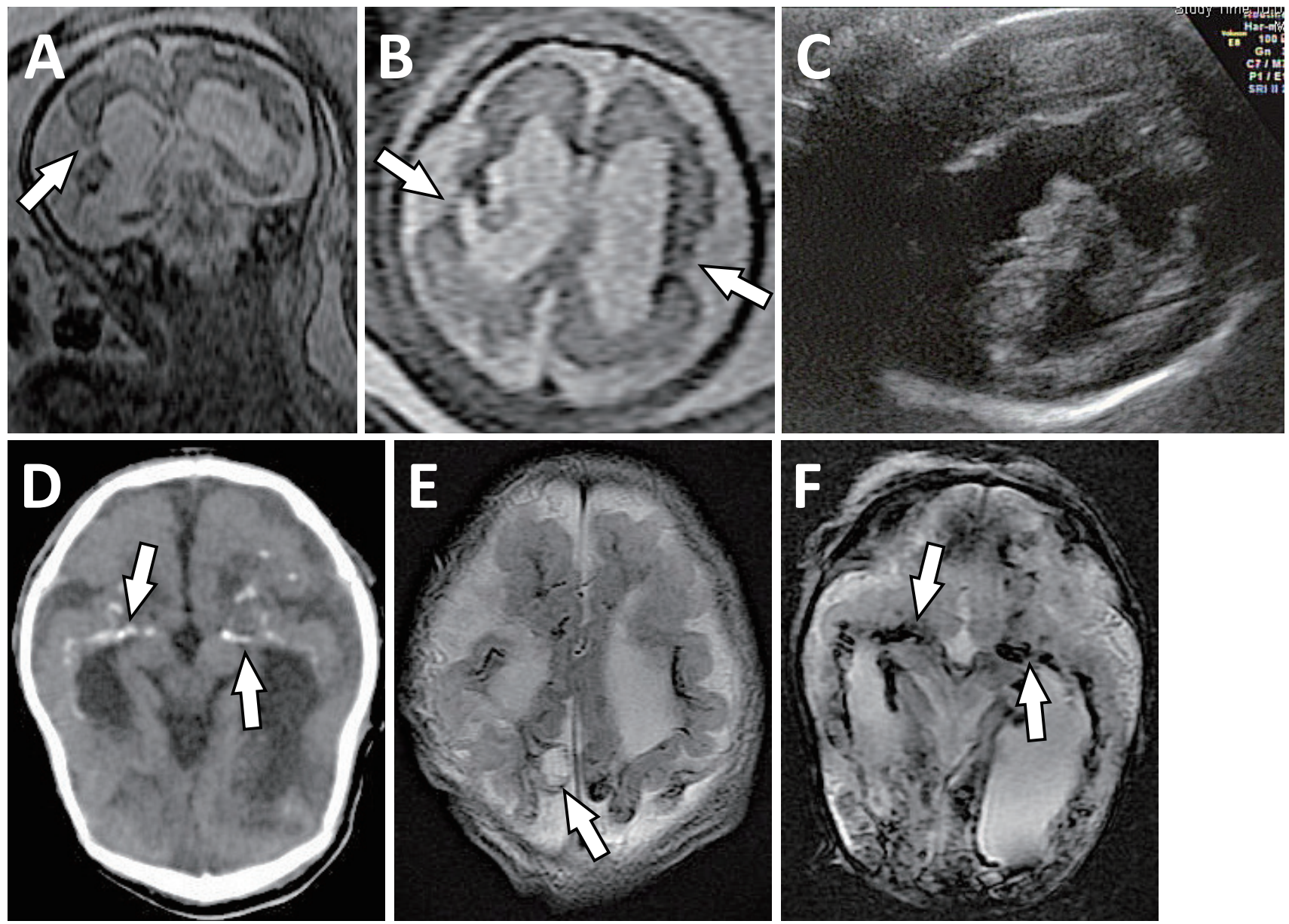

Fig. 2. The results of diagnostic imaging at pre- and postnatal period of Case 2. A, B, C: Prenatal transabdominal ultrasound and magnetic resonance images. A, B: Cyst formation and irregular thinning of the cerebrum, as seen on magnetic resonance imaging (T2-weighted images) at 32 weeks 0 days. The ependyma merges with the pia mater in the area of cerebral parenchymal defects (arrows). Hypointensities are present along the ventricular walls and brain surface on the T2-weighted images (arrows). C. Ventriculomegaly, as seen on transabdominal ultrasound at 36 weeks 5 days. D, E, F: Postnatal magnetic resonance and computed tomography images. D, Calcification around the lateral ventricles, as seen on computed tomography on postnatal day 10 (arrows). E, F: Cyst formation in the cerebrum, as seen on magnetic resonance imaging (T2-weighted images) on postnatal day 10 (arrows). Hypointensities on T2-weighted images appear in the same regions as the calcification seen on computed tomography (arrows).

COL4A1 gene (COL4A1 c.4843G > A [p.Glu1615Lys]). She was diagnosed with symptomatic epilepsy, and phenobarbital was started on postnatal day 17. She was transferred to a local hospital on postnatal day 60 . She also had right corneal opacity, right optic atrophy, and left ocular hypoplasia, and she underwent gastrostomy tube placement for gastroesophageal reflux and laryngotracheal separation for laryngomalacia.

\section{DISCUSSION}

Schizencephaly was named by Yakovlev et al. in 1946 based on the morphological features of autopsy specimens. ${ }^{1}$ Diagnostic criteria for schizencephaly were subsequently proposed by Miller et al. in 1984, based on characteristics observed on diagnostic images. ${ }^{7}$ Poren- cephaly was first reported by Heschl et al. in 1859, and the diagnosis was based on autopsy findings of cerebral clefts and cysts communicating with the subarachnoid space and lateral ventricles. ${ }^{8}$ However, no studies to date have compared the histopathological and radiographic features of schizencephaly and porencephaly, and no clear method for differentiating between the two disorders has yet been established. Our experiences with the two cases reported here, however, suggest that MRI may be a useful diagnostic tool for pre- and postnatal differentiation. Ultrasound observation of the fetal cranium enables diagnosis of ventriculomegaly, cerebral parenchymal defects, and large clefts; however, the marked effects of bone artifacts, particularly from the second trimester on, prevent detailed examination. Furthermore, 
the similar incidence of unilateral and bilateral clefts in schizencephaly ${ }^{9}$ and differences in cleft size between individuals make it difficult to differentiate schizencephaly from porencephaly based on cleft size alone. The neurologic symptoms and developmental outcome of children with schizencephaly differ in the extent of cortex involved in the cleft. Bilateral open-lip type defect is the poor prognostic factor. ${ }^{10}$ The prenatal imaging variation of defect size and locations may have important implications for counseling and suggesting treatment options for these patients. ${ }^{11}$ This was our experience with Case 1: large cleft size precluded definitive differentiation of schizencephaly from porencephaly by prenatal ultrasound examination, but we were able to make the prenatal diagnosis of schizencephaly by MRI, as it allowed us to confirm the absence of cystic changes in the cerebrum. We were not able to confirm the presence of clefts lined by gray matter that extend from the brain surface to the lateral ventricules before birth, owing to the low resolution of fetal MRI imaging. Postnatal MRI observation of abnormal gray matter lining the clefts was useful in confirming the diagnosis of schizencephaly.

Differences in morphological features between schizencephaly and porencephaly are thought to arise due to the occurrence of destructive changes at different stages of cerebral cortex formation. ${ }^{3,6}$ Causes of these destructive changes in the cerebral mantle include: maternal-fetal transmission of infections such as CMV and toxoplasma; monozygotic twinning; and maternal cardiac arrest or abdominal trauma. ${ }^{12,13}$ Both of the present cases were negative for CMV infection, and the maternal medical histories showed no symptoms indicative of maternal-fetal transmission or physical trauma. However, neonatal MRI performed immediately after birth indicated that hemorrhagic changes had occurred during the fetal period; thus, the cause of disease in both of the present cases was presumed to be fetal intracranial hemorrhage.

A relationship between intracranial hemorrhage and mutation of the procollagen alpha-1 (IV) (COL4AI) gene has recently been reported. ${ }^{6}$ The COL4Al gene codes for the alpha- 1 chain of the heterotrimeric type IV collagen molecule, a primary component of the vascular basement membrane. ${ }^{14}$ Changes in the vascular walls due to this genetic mutation result in abnormalities in the brain, eyeballs, muscles, kidneys, and other organs. ${ }^{15} \mathrm{In}$ mice, COL4A1 mutation causes ocular dysgenesis, cortical dysplasia, cerebral hemorrhage, porencephaly, and myopathy. ${ }^{16,17}$ Approximately $20 \%$ of patients with schizencephaly or porencephaly have a COL $4 A 1$ mutation. ${ }^{6}$ Genetic testing in Case 2 of the present study identified a heterozygous missense mutation in the COL4Al gene (COL4A1 c.4843G > A [p.Glu1615Lys]). Thus, porencephaly was likely caused by fetal intracranial hemorrhage due to the COL4A1 mutation.

Porencephaly does not appear to result from $\mathrm{CO}$ L4A1 mutation-related destructive changes and intracranial hemorrhage alone; many contributing factors in addition to genetic mutation are believed to be involved. ${ }^{16}$ However, the many reports of familial recurrence of schizencephaly and porencephaly, and of an autosomal dominant pattern of inheritance of porencephaly, ${ }_{18}^{18}$ indicate that genetic factors are important to the disease etiologies. Familial porencephaly likely involves vascular wall weakening due to genetic mutation; therefore, delivery by cesarean section is recommended to avoid injury at the time of birth ${ }^{16}$. In the present porencephaly case, further testing was required to identify genetic mutations and facilitate appropriate postpartum counseling for the parents.

In conclusion, cases of schizencephaly and porencephaly were described. Differentiation between schizencephaly and porencephaly is challenging when the schizencephalic clefts are large. However, using MRI to confirm the presence or absence of cystic changes or abnormal gray matter lining the clefts aids differentiation.

The authors declare no conflict of interest.

\section{REFERENCES}

1 Yakovlev PI, Wadsworth RC. Schizencephalies; a study of the congenital clefts in the cerebral mantle; clefts with fused lips. J Neuropathol Exp Neurol. 1946;5:116-30. PMID: 21026933.

2 Yakovlev PI, Wadsworth RC. Schizencephalies; a study of the congenital clefts in the cerebral mantle; clefts with hydrocephalus and lips separated. J Neuropathol Exp Neurol. 1946;5:169-206. PMID: 20993391.

3 Eller KM, Kuller JA. Fetal porencephaly: a review of etiology, diagnosis, and prognosis. Obstet Gynecol Surv. 1995;50:6847. PMID: 7478420.

4 Friede RL. Porencephaly, Hydranencephaly, Multicystic Encephalopathy. Developmental Neuropathology. 2nd (ed). Springer-Verlag Berlin Heidelberg. 1989, pp 28-43. DOI: 10.1007/978-3-642-73697-1_3.

5 Oh KY, Kennedy AM, Frias AE, Jr., Byrne JL. Fetal schizencephaly: pre- and postnatal imaging with a review of the clinical manifestations. Radiographics. 2005;25:647-57. PMID: 15888615.

6 Yoneda Y, Haginoya K, Kato M, Osaka H, Yokochi K, Arai $\mathrm{H}$, et al. Phenotypic spectrum of COL4A1 mutations: porencephaly to schizencephaly. Ann Neurol. 2013;73:48-57. PMID: 23225343.

7 Miller GM, Stears JC, Guggenheim MA, Wilkening GN. Schizencephaly: a clinical and CT study. Neurology. 1984;34:997-1001. PMID: 6540398.

8 Heschl R. Gehirndefect und Hydrocephalus. Vierteljahrschrift fur praktikale Heikunde Prague.1859;61:59-74. German. 
9 Hayashi N, Tsutsumi Y, Barkovich AJ. Morphological features and associated anomalies of schizencephaly in the clinical population: detailed analysis of MR images. Neuroradiology. 2002;44:418-27. PMID: 12012128.

10 Packard AM, Miller VS, Delgnado MR. Schizencephaly: correlations of clinical and radiologic features. 1997;48:1427-34. PMID: 9153485.

11 Nabavizadeh SA, Zarnow D, Bilaniuk LT, Schwartz ES, Zimmerman RA, Vossough A. Correlation of prenatal and postnatal MRI findings in schizencephaly. AJNR Am J Neuroradiol. 2014;35:1418-24. PMID: 24610904.

12 Govaert P. Prenatal stroke. Semin Fetal Neonatal Med. 2009;14:250-66. PMID: 19664975.

13 Moinuddin A, McKinstry RC, Martin KA, Neil JJ. Intracranial hemorrhage progressing to porencephaly as a result of congenitally acquired cytomegalovirus infection-an illustrative report. Prenatal Diagnosis. 2003;23:797-800. PMID: 14558022.
14 Khoshnoodi J, Pedchenko V, Hudson BG. Mammalian collagen IV. Microsc Res Tech. 2008;71:357-70. PMID: 18219669.

15 de Vries LS, Mancini GM. Intracerebral hemorrhage and $C O$ L4A1 and COL4A2 mutations, from fetal life into adulthood. Ann Neurol. 2012;71:439-41. PMID: 22447691.

16 Gould DB, Phalan FC, Breedveld GJ, van Mil SE, Smith RS, Schimenti JC, et al. Mutations in Col4al cause perinatal cerebral hemorrhage and porencephaly. Science. 2005;308:1167171. PMID: 15905400.

17 Labelle-Dumais C, Dilworth DJ, Harrington EP, de Leau M, Lyons D, Kabaeva Z, et al. COL4A1 mutations cause ocular dysgenesis, neuronal localization defects, and myopathy in mice and Walker-Warburg syndrome in humans. PLoS Genetics. 2011;7:e1002062. PMID: 21625620.

18 de Vries LS, Koopman C, Groenendaal F, Van Schooneveld $\mathrm{M}$, Verheijen FW, Verbeek E, et al. COLAA1 mutation in two preterm siblings with antenatal onset of parenchymal hemorrhage. Ann Neurol. 2009;65:12-18. PMID: 19194877. 